\title{
Local convergence of the Gauss-Newton-Kurchatov method under generalized Lipschitz conditions
}

\begin{abstract}
Shakhno S.M. ${ }^{\bowtie}$, Yarmola H.P.
We investigate the local convergence of the Gauss-Newton-Kurchatov method for solving nonlinear least squares problems. This method is a combination of Gauss-Newton and Kurchatov methods and it is used for problems with the decomposition of the operator. The convergence analysis of the method is performed under the generalized Lipshitz conditions. The conditions of convergence, radius and the convergence order of the considered method are established. Given numerical examples confirm the theoretical results.

Key words and phrases: Gauss-Newton-Kurchatov method, local convergence, Fréchet derivative, divided difference, generalized Lipschitz condition, convergence domain.
\end{abstract}

Ivan Franko Lviv National University, 1 Universytetska str., 79000, Lviv, Ukraine

$凶$ Corresponding author

E-mail: stepan. shakhno@lnu.edu.ua (Shakhno S.M.), halyna.yarmola@lnu.edu.ua (Yarmola H.P.)

\section{Introduction}

One of the important problems in Computational Mathematics is finding numerical solutions of nonlinear least squares problems. They arise while solving overdetermined systems of nonlinear equations, parameter estimation of physical processes by measurement results, constructing nonlinear regression models for solving engineering problems.

The classical formulation of the nonlinear least squares problem looks like this $[1,2,7,10]$ :

$$
\min _{x \in D} \frac{1}{2} F(x)^{T} F(x) \text {. }
$$

Here residual function $F: D \subseteq \mathbb{R}^{n} \rightarrow \mathbb{R}^{m}, m \geq n$, is nonlinear by $x, D$ is an open convex domain, $F$ is a continuously differentiable function. The most used technique for solving of (1) is Gauss-Newton's method $[1,2,7,10]$

$$
x_{k+1}=x_{k}-\left(F^{\prime}\left(x_{k}\right)^{T} F^{\prime}\left(x_{k}\right)\right)^{-1} F^{\prime}\left(x_{k}\right)^{T} F\left(x_{k}\right), \quad k=0,1, \ldots
$$

Difference methods are also often used $[11,12,14]$. One of them is the Kurchatov type method $[12,14]$

$$
L_{k}=F\left(2 x_{k}-x_{k-1}, x_{k-1}\right), \quad x_{k+1}=x_{k}-\left(L_{k}^{T} L_{k}\right)^{-1} L_{k}^{T} F\left(x_{k}\right), \quad k=0,1, \ldots
$$

Here $F^{\prime}\left(x_{k}\right)$ is a Fréchet derivative of $F(x) ; F\left(2 x_{k}-x_{k-1}, x_{k-1}\right)$ is a divided difference of the first order of function $F(x)$ (see $[18,19]$ ) at points $2 x_{k}-x_{k-1}, x_{k-1} ; x_{0}, x_{-1}$ are given starting points. 
Let us consider the nonlinear least squares problem with decomposition of operator [15-17]

$$
\min _{x \in D} \frac{1}{2}(F(x)+G(x))^{T}(F(x)+G(x)),
$$

where the residual function $F+G: D \subseteq \mathbb{R}^{n} \rightarrow \mathbb{R}^{m}, m \geq n$, is nonlinear by $x, D$ is an open convex domain, $F$ is a continuously differentiable function, $G$ is a continuous function, differentiability of which, in general, is not required. For numerical solving of (4) one can use difference and combined methods. The classical Gauss-Newton method cannot be applied to solving this problem.

Combination of (2) and (3) gives the Gauss-Newton-Kurchatov method for finding the solution of the problem (4)

$$
\begin{aligned}
A_{k} & =F^{\prime}\left(x_{k}\right)+G\left(2 x_{k}-x_{k-1}, x_{k-1}\right), \\
x_{k+1} & =x_{k}-\left(A_{k}^{T} A_{k}\right)^{-1} A_{k}^{T}\left(F\left(x_{k}\right)+G\left(x_{k}\right)\right), \quad k=0,1, \ldots .
\end{aligned}
$$

We proposed this method in [13]. Another combined methods are considered in [15-17].

For $m=n$, the problem (4) turns into a system of nonlinear equations $F(x)+G(x)=0$. This problem and methods for its solving were studied in $[1-6,8,9]$.

In this paper, we provide the local convergence analysis of the Gauss-Newton-Kurchatov method (5). There are two main approaches to the study the convergence of iterative methods: a local and semilocal convergence analysis. In the first case, the existence of a solution $x^{*}$ is assumed. Then, based on the information around a solution, it is found the radius of convergence ball with a center in solution and asserted that the sequence, generated by an iterative method, is well defined, remains in convergence ball, and converges to this solution. In the second case, based on the information around an initial point $x_{0}$, it is found the radius of convergence ball with a center in initial point and asserted that the sequence, generated by iterative methods, is well defined, remains in convergence ball, and converges to the solution that contains in this ball. Moreover, theorems of both types usually include error estimates on $\left\|x_{k}-x^{*}\right\|$. The semilocal convergence theorems additionally include error estimates on $\left\|x_{k+1}-x_{k}\right\|$.

\section{Local convergence analysis}

We give a local convergence analysis of the Gauss-Newton-Kurchatov method (5) under generalized Lipschitz conditions. These conditions were proposed in [20].

Let us, at first, consider some auxiliary lemmas $[15,20]$ needed to obtain the main results.

Lemma 1. Put

$$
e(t)=\int_{0}^{t} E(u) d u
$$

where $E$ is integrable and positive nondecreasing function on $[0, T]$. The function $e(t)$ is monotonically increasing with respect to $t$ on $[0, T]$.

Lemma 2. Put

$$
h(t)=\frac{1}{t} \int_{0}^{t} H(u) d u,
$$

where $H$ is integrable and positive nondecreasing function on $[0, T]$. The function $h(t)$ is nondecreasing with respect to $t$ on $(0, T]$. 
Lemma 3. Put

$$
s(t)=\frac{1}{t^{2}} \int_{0}^{t} S(u) u d u,
$$

where $S$ is integrable and positive nondecreasing function on $[0, T]$. The function $s(t)$ is nondecreasing with respect to $t$ on $(0, T]$.

Sufficient conditions and the rate of local convergence of the iterative process (5) are defined in such a theorem. We use the Euclidean norm, for which $\|A-B\|=\left\|A^{T}-B^{T}\right\|$, with $A, B \in \mathbb{R}^{m \times n}$, is fulfilled. Denote

$$
\Omega\left(x^{*}, \tau\right)=\left\{x \in D:\left\|x-x^{*}\right\|<\tau\right\}, \quad x^{*} \in D, \tau>0, \quad A_{*}=F^{\prime}\left(x^{*}\right)+G\left(x^{*}, x^{*}\right) .
$$

Theorem 1. Let $F+G: D \subseteq \mathbb{R}^{n} \rightarrow \mathbb{R}^{m}$ be continuous on an open convex subset $D$, and $F$ is a continuously differentiable function, $G$ is a continuous function. Suppose that the problem (4) has a solution $x^{*} \in D$, and the inverse operation $\left(A_{*}^{T} A_{*}\right)^{-1}$ exists, such that $\left\|\left(A_{*}^{T} A_{*}\right)^{-1}\right\| \leq B$.

On the subset $D$, the Fréchet derivative $F^{\prime}$ satisfies the radius Lipschitz condition with $L$ average

$$
\left\|F^{\prime}(x)-F^{\prime}\left(x^{\tau}\right)\right\| \leq \int_{\tau \rho(x)}^{\rho(x)} L(u) d u, \quad x^{\tau}=x^{*}+\tau\left(x-x^{*}\right), \quad 0 \leq \tau \leq 1,
$$

the function $G$ has the first- and second-order divided difference, and

$$
\begin{gathered}
\|G(x, y)-G(u, v)\| \leq \int_{0}^{\|x-u\|+\|y-v\|} M(u) d u, \\
\|G(u, x, y)-G(v, x, y)\| \leq \int_{0}^{\|u-v\|} N(t) d t,
\end{gathered}
$$

for all $x, y, u, v \in D, \rho(x)=\left\|x-x^{*}\right\|, L, M$ and $N$ are positive nondecreasing functions on $[0,2 R], R>0$.

Furthermore,

$$
\left\|F\left(x^{*}\right)+G\left(x^{*}\right)\right\| \leq \eta, \quad\left\|F^{\prime}\left(x^{*}\right)+G\left(x^{*}, x^{*}\right)\right\| \leq \alpha, \quad \frac{\eta}{R}\left[\int_{0}^{R} L(u) d u+\int_{0}^{2 R} M(u) d u\right]<1,
$$

and $\Omega\left(x^{*}, 3 r_{*}\right) \subseteq D$, where $r_{*}$ is the unique positive zero of the function $q$ given by

$$
\begin{aligned}
q(r)= & B\left[\left[\alpha+\int_{0}^{r} L(u) d u+\int_{0}^{2 r} M(u) d u+2 r \int_{0}^{2 r} N(u) d u\right]\right. \\
& \times\left[\frac{1}{r} \int_{0}^{r} L(u) u d u+\int_{0}^{r} M(u) d u+2 r \int_{0}^{2 r} N(u) d u\right] \\
+ & {\left[2 \alpha+\int_{0}^{r} L(u) d u+\int_{0}^{2 r} M(u) d u+2 r \int_{0}^{2 r} N(u) d u\right] } \\
& \times\left[\int_{0}^{r} L(u) d u+\int_{0}^{2 r} M(u) d u+2 r \int_{0}^{2 r} N(u) d u\right] \\
+ & \left.\frac{1}{r}\left[\int_{0}^{r} L(u) d u+\int_{0}^{2 r} M(u) d u+2 r \int_{0}^{2 r} N(u) d u\right] \eta\right]-1 .
\end{aligned}
$$

Then, for $x_{0}, x_{-1} \in \Omega\left(x^{*}, r_{*}\right)$, the iterative process $\left\{x_{k}\right\}, k=0,1, \ldots$, generated by (5), is well defined, remains in $\Omega\left(x^{*}, r_{*}\right)$, and converges to $x^{*}$. Moreover, the following error estimates hold for all $k \geq 0$ :

$$
\left\|x_{k+1}-x^{*}\right\| \leq C_{1}\left\|x_{k}-x^{*}\right\|+C_{2}\left\|x_{k}-x_{k-1}\right\|^{2}+C_{3}\left\|x_{k}-x^{*}\right\|^{2}+C_{4}\left\|x_{k}-x^{*}\right\|\left\|x_{k}-x_{k-1}\right\|^{2},
$$


where

$$
\begin{gathered}
C_{1}=g\left(r_{*}\right) \frac{\eta}{r_{*}}\left(\int_{0}^{r_{*}} L(u) d u+\int_{0}^{2 r_{*}} M(u) d u\right), \quad C_{2}=g\left(r_{*}\right) \frac{\eta}{2 r_{*}} \int_{0}^{2 r_{*}} N(u) d u, \\
C_{3}=\frac{g\left(r_{*}\right) T\left(r_{*}\right)}{r_{*}}\left(\frac{1}{r_{*}} \int_{0}^{r_{*}} L(u) u d u+\int_{0}^{r_{*}} M(u) d u\right), \quad C_{4}=\frac{g\left(r_{*}\right) T\left(r_{*}\right)}{2 r_{*}} \int_{0}^{2 r_{*}} N(u) d u, \\
g(r)=B(1-B[T(r)+\alpha][T(r)-\alpha])^{-1}, \\
T(r)=\alpha+\int_{0}^{r} L(u) d u+\int_{0}^{2 r} M(u) d u+2 r \int_{0}^{2 r} N(u) d u .
\end{gathered}
$$

Proof. According to L'Hospital's rule we get

$$
\lim _{r \rightarrow 0} \frac{1}{r} \int_{0}^{r} L(u) d u=\lim _{r \rightarrow 0} \frac{L(r)}{1}=L(0), \quad \lim _{r \rightarrow 0} \frac{1}{r} \int_{0}^{2 r} M(u) d u=\lim _{r \rightarrow 0} \frac{2 M(2 r)}{1}=2 M(0) .
$$

Then for sufficiently small $\eta$ we have $q(0)=B(L(0)+2 M(0)) \eta-1<0$. Taking into account Lemmas $1-3$ we can prove, that $q(r)$ is monotonically increasing on $(0, R]$. With a sufficiently large $R$ inequality $q(R)>0$ holds. By the intermediate value theorem, the function $q$ has on $(0, R)$ a positive zero denoted by $r_{*}$. Moreover $q^{\prime}(R) \geq 0$ for $R \geq 0$. Therefore, this zero is unique on $(0, R)$.

Denote $\theta_{k}=\left\|x_{k}-x_{k-1}\right\|$. Let $k=0$. Then we obtain the following estimation

$$
\begin{aligned}
\left\|I-\left(A_{*}^{T} A_{*}\right)^{-1} A_{0}^{T} A_{0}\right\| \\
\\
=\left\|\left(A_{*}^{T} A_{*}\right)^{-1}\left(A_{*}^{T}\left(A_{*}-A_{0}\right)+\left(A_{*}^{T}-A_{0}^{T}\right)\left(A_{0}-A_{*}\right)+\left(A_{*}^{T}-A_{0}^{T}\right) A_{*}\right)\right\| \\
\leq B\left(\alpha\left\|A_{*}-A_{0}\right\|+\left\|A_{*}^{T}-A_{0}^{T}\right\|\left\|A_{0}-A_{*}\right\|+\alpha\left\|A_{*}^{T}-A_{0}^{T}\right\|\right) .
\end{aligned}
$$

Using conditions (6)-(8), we get

$$
\begin{aligned}
\left\|A_{0}-A_{*}\right\|= & \| F^{\prime}\left(x_{0}\right)-F^{\prime}\left(x^{*}\right)+G\left(2 x_{0}-x_{-1}, x_{-1}\right)-G\left(x_{0}, x_{-1}\right) \\
& \quad+G\left(x_{0}, x_{-1}\right)-G\left(x_{0}, x_{0}\right)+G\left(x_{0}, x_{0}\right)-G\left(x_{*}, x_{*}\right) \| \\
\leq & \left\|F^{\prime}\left(x_{0}\right)-F^{\prime}\left(x^{*}\right)\right\|+\left\|G\left(x_{0}, x_{0}\right)-G\left(x_{*}, x_{*}\right)\right\| \\
& \quad+\left\|\left(G\left(x_{0}, x_{-1}, x_{0}\right)-G\left(2 x_{0}-x_{-1}, x_{-1}, x_{0}\right)\right)\left(x_{0}-x_{-1}\right)\right\| \\
\leq & \int_{0}^{\rho_{0}} L(u) d u+\int_{0}^{2 \rho_{0}} M(u) d u+\left\|x_{0}-x_{-1}\right\| \int_{0}^{\theta_{0}} N(u) d u .
\end{aligned}
$$

Then from (10), (11) and definition of $r_{*}$ we get

$$
\begin{aligned}
&\left\|I-\left(A_{*}^{T} A_{*}\right)^{-1} A_{0}^{T} A_{0}\right\| \leq B {\left[2 \alpha+\int_{0}^{\rho_{0}} L(u) d u+\int_{0}^{2 \rho_{0}} M(u) d u+\left\|x_{0}-x_{-1}\right\| \int_{0}^{\theta_{0}} N(u) d u\right] } \\
& \times\left[\int_{0}^{\rho_{0}} L(u) d u+\int_{0}^{2 \rho_{0}} M(u) d u+\left\|x_{0}-x_{-1}\right\| \int_{0}^{\theta_{0}} N(u) d u\right] \\
& \leq B {\left[2 \alpha+\int_{0}^{r_{*}} L(u) d u+\int_{0}^{2 r_{*}} M(u) d u+2 r_{*} \int_{0}^{2 r_{*}} N(u) d u\right] } \\
& \times {\left[\int_{0}^{r_{*}} L(u) d u+\int_{0}^{2 r_{*}} M(u) d u+2 r_{*} \int_{0}^{2 r_{*}} N(u) d u\right]<1 . }
\end{aligned}
$$


According to Banach lemma of invertible operator $[1,10]$ and (12), it follows that $\left(A_{0}^{T} A_{0}\right)^{-1}$ exists and

$$
\begin{aligned}
&\left\|\left(A_{0}^{T} A_{0}\right)^{-1}\right\| \leq g_{0}=B(1-B {\left[2 \alpha+\int_{0}^{\rho_{0}} L(u) d u+\int_{0}^{2 \rho_{0}} M(u) d u+\left\|x_{0}-x_{-1}\right\| \int_{0}^{\theta_{0}} N(u) d u\right] } \\
& \times {\left.\left[\int_{0}^{\rho_{0}} L(u) d u+\int_{0}^{2 \rho_{0}} M(u) d u+\left\|x_{0}-x_{-1}\right\| \int_{0}^{\theta_{0}} N(u) d u\right]\right)^{-1} } \\
& \leq g\left(r_{*}\right)=B\left(1-B\left[2 \alpha+\int_{0}^{r_{*}} L(u) d u+\int_{0}^{2 r_{*}} M(u) d u+2 r_{*} \int_{0}^{2 r_{*}} N(u) d u\right]\right. \\
&\left.\times\left[\int_{0}^{r_{*}} L(u) d u+\int_{0}^{2 r_{*}} M(u) d u+2 r_{*} \int_{0}^{2 r_{*}} N(u) d u\right]\right)^{-1} .
\end{aligned}
$$

Hence, $x_{1}$ is well defined. Next, we can write

$$
\begin{aligned}
\left\|x_{1}-x^{*}\right\| & =\left\|x_{0}-x^{*}-\left(A_{0}^{T} A_{0}\right)^{-1}\left(A_{0}^{T}\left(F\left(x_{0}\right)+G\left(x_{0}\right)\right)-A_{*}^{T}\left(F\left(x^{*}\right)+G\left(x^{*}\right)\right)\right)\right\| \\
\leq\left\|-\left(A_{0}^{T} A_{0}\right)^{-1}\right\| \| & -A_{0}^{T}\left(A_{0}-\int_{0}^{1} F^{\prime}\left(x^{*}+t\left(x_{0}-x^{*}\right)\right) d t-G\left(x_{0}, x^{*}\right)\right)\left(x_{0}-x^{*}\right) \\
& +\left(A_{0}^{T}-A_{*}^{T}\right)\left(F\left(x^{*}\right)+G\left(x^{*}\right)\right) \| .
\end{aligned}
$$

Thus, by conditions (6)-(8) and inequalities

$$
\begin{aligned}
\| A_{0}-\int_{0}^{1} F^{\prime}\left(x^{*}\right. & \left.+t\left(x_{0}-x^{*}\right)\right) d t-G\left(x_{0}, x^{*}\right) \| \\
& =\left\|F^{\prime}\left(x_{0}\right)-\int_{0}^{1} F^{\prime}\left(x^{*}+t\left(x_{0}-x^{*}\right)\right) d t+G\left(2 x_{0}-x_{-1}, x_{-1}\right)-G\left(x_{0}, x^{*}\right)\right\| \\
& \leq \int_{0}^{1} \int_{\tau \rho_{0}}^{\rho_{0}} L(u) d u d \tau+\int_{0}^{\rho_{0}} M(u) d u+\left\|x_{0}-x_{-1}\right\| \int_{0}^{\theta_{0}} N(u) d u \\
& \leq \frac{1}{\rho_{0}} \int_{0}^{\rho_{0}} L(u) u d u+\int_{0}^{\rho_{0}} M(u) d u+\left\|x_{0}-x_{-1}\right\| \int_{0}^{\theta_{0}} N(u) d u,
\end{aligned}
$$

and

$$
\left\|A_{0}\right\| \leq\left\|A_{*}\right\|+\left\|A_{0}-A_{*}\right\| \leq \alpha+\int_{0}^{\rho_{0}} L(u) d u+\int_{0}^{2 \rho_{0}} M(u) d u+\left\|x_{0}-x_{-1}\right\| \int_{0}^{\theta_{0}} N(u) d u
$$

we obtain

$$
\begin{aligned}
\left\|x_{1}-x^{*}\right\| \leq g_{0}[ & {\left[\alpha+\int_{0}^{\rho_{0}} L(u) d u+\int_{0}^{2 \rho_{0}} M(u) d u+\left\|x_{0}-x_{-1}\right\| \int_{0}^{\theta_{0}} N(u) d u\right] } \\
& \times\left[\frac{1}{\rho_{0}} \int_{0}^{\rho_{0}} L(u) u d u+\int_{0}^{\rho_{0}} M(u) d u+\int_{0}^{\theta_{0}} N(u) d u\left\|x_{0}-x_{-1}\right\|\right]\left\|x_{0}-x^{*}\right\| \\
+ & {\left.\left[\int_{0}^{\rho_{0}} L(u) d u+\int_{0}^{2 \rho_{0}} M(u) d u+\left\|x_{0}-x_{-1}\right\| \int_{0}^{\theta_{0}} N(u) d u\right]\right] } \\
\leq & g\left(r_{*}\right)\left[\left[\alpha+\int_{0}^{r_{*}} L(u) d u+\int_{0}^{2 r_{*}} M(u) d u+2 r_{*} \int_{0}^{2 r_{*}} N(u) d u\right]\right. \\
& \times\left[\frac{1}{r_{*}} \int_{0}^{r_{*}} L(u) u d u+\int_{0}^{r_{*}} M(u) d u+2 r_{*} \int_{0}^{2 r_{*}} N(u) d u\right] \\
+ & \frac{\eta}{r_{*}}\left[\int_{0}^{r_{*}} L(u) d u+\int_{0}^{2 r_{*}} M(u) d u+2 r_{*}^{2 r_{*}} N(u) d u\right] r_{0} .
\end{aligned}
$$


So, $x_{1} \in \Omega\left(x^{*}, r_{*}\right)$ and estimate (9) is true for $k=0$.

Let us suppose, that $x_{k} \in \Omega\left(x^{*}, r_{*}\right)$ for $k \geq 0$ and estimate (9) holds. Let us prove, that $x_{k+1} \in \Omega\left(x^{*}, r_{*}\right)$ and estimate (9) holds.

Using conditions (6)-(8), we get

$$
\begin{aligned}
\left\|I-\left(A_{*}^{T} A_{*}^{T}\right)^{-1} A_{k}^{T} A_{k}\right\| \leq & B\left[2 \alpha+\int_{0}^{\rho_{k}} L(u) d u+\int_{0}^{2 \rho_{k}} M(u) d u+\left\|x_{k}-x_{k-1}\right\| \int_{0}^{\theta_{k}} N(u) d u\right] \\
& \times\left[\int_{0}^{\rho_{k}} L(u) d u+\int_{0}^{2 \rho_{k}} M(u) d u+\left\|x_{k}-x_{k-1}\right\| \int_{0}^{\theta_{k}} N(u) d u\right] \\
\leq & B\left[2 \alpha+\int_{0}^{r_{*}} L(u) d u+\int_{0}^{2 r_{*}} M(u) d u+2 r_{*} \int_{0}^{2 r_{*}} N(u) d u\right] \\
& \times\left[\int_{0}^{r_{*}} L(u) d u+\int_{0}^{2 r_{*}} M(u) d u+2 r_{*} \int_{0}^{2 r_{*}} N(u) d u\right]<1 .
\end{aligned}
$$

Thus, $\left(A_{k}^{T} A_{k}\right)^{-1}$ exists and

$$
\begin{aligned}
\left\|\left(A_{k}^{T} A_{k}\right)^{-1}\right\| \leq g_{k}= & B\left[1-B\left[2 \alpha+\int_{0}^{\rho_{k}} L(u) d u+\int_{0}^{2 \rho_{k}} M(u) d u+\left\|x_{k}-x_{k-1}\right\| \int_{0}^{\theta_{k}} N(u) d u\right]\right. \\
& \left.\times\left[\int_{0}^{\rho_{k}} L(u) d u+\int_{0}^{2 \rho_{k}} M(u) d u+\left\|x_{k}-x_{k-1}\right\| \int_{0}^{\theta_{k}} N(u) d u\right]\right] \leq g\left(r_{*}\right) .
\end{aligned}
$$

Hence,

$$
\begin{aligned}
\left\|x_{k+1}-x^{*}\right\| \leq g_{k} & {\left[\left[\alpha+\int_{0}^{\rho_{k}} L(u) d u+\int_{0}^{2 \rho_{k}} M(u) d u+\left\|x_{k}-x_{k-1}\right\| \int_{0}^{\theta_{k}} N(u) d u\right]\right.} \\
& \times\left[\frac{1}{\rho_{k}} \int_{0}^{\rho_{k}} L(u) u d u+\int_{0}^{\rho_{k}} M(u) d u+\left\|x_{k}-x_{k-1}\right\| \int_{0}^{\theta_{k}} N(u) d u\right]\left\|x_{k}-x^{*}\right\| \\
+ & \left.\eta\left[\int_{0}^{\rho_{k}} L(u) d u+\int_{0}^{2 \rho_{k}} M(u) d u+\left\|x_{k}-x_{k-1}\right\| \int_{0}^{\theta_{k}} N(u) d u\right]\right] \\
\leq & g\left(r_{*}\right)\left[\left[\alpha+\int_{0}^{r_{*}} L(u) d u+\int_{0}^{2 r_{*}} M(u) d u+2 r_{*} \int_{0}^{2 r_{*}} N(u) d u\right]\right. \\
& \times\left[\frac{1}{r_{*}} \int_{0}^{r_{*}} L(u) u d u+\int_{0}^{r_{*}} M(u) d u+2 r_{*} \int_{0}^{2 r_{*}} N(u) d u\right] \\
+ & \left.\frac{\eta}{r_{*}}\left[\int_{0}^{r_{*}} L(u) d u+\int_{0}^{2 r_{*}} M(u) d u+2 r_{*} \int_{0}^{2 r_{*}} N(u) d u\right]\right] r_{*} .
\end{aligned}
$$

and $x_{k+1} \in \Omega\left(x^{*}, r_{*}\right)$.

Thus, iterative process (5) is well defined, $x_{k+1} \in \Omega\left(x^{*}, r_{*}\right)$ for $k \geq 0$ and estimate (9) holds for each $k \geq 0$.

Let us prove that $x_{k} \rightarrow x^{*}$ for $k \rightarrow \infty$. Define functions $a, b$ on $\left[0, r_{*}\right]$ by

$$
a(r)=C_{1}+C_{3} r+4 C_{4} r^{2}, \quad b(r)=4 C_{2} r .
$$

According to the choice of $r_{*}$, we get

$$
a\left(r_{*}\right) \geq 0, \quad b\left(r_{*}\right) \geq 0, \quad a\left(r_{*}\right)+b\left(r_{*}\right)=1 .
$$

Using the estimate (9), the definition of functions $a, b$ and constants $C_{i}, i=1,2,3,4$, we get

$$
\left\|x_{k+1}-x^{*}\right\| \leq a\left(r_{*}\right)\left\|x_{k}-x^{*}\right\|+b\left(r_{*}\right)\left\|x_{k-1}-x^{*}\right\| .
$$

According to the proof in [1,2], under the conditions (13) and (14), the sequence $\left\{x_{k}\right\}$ converges to $x^{*}$ for $k \rightarrow \infty$. 
Corollary 1. The convergence order of the iterative method (5) with zero residual is quadratic.

Proof. If $\eta=0$, we have the nonlinear least squares problem with zero residual in the solution. In this case constants $C_{1}=0, C_{2}=0$, and the estimate (9) reduces to

$$
\begin{aligned}
\left\|x_{k+1}-x^{*}\right\| & \leq C_{3}\left\|x_{k}-x^{*}\right\|^{2}+C_{4}\left\|x_{k}-x^{*}\right\|\left\|x_{k-1}-x_{k}\right\|^{2} \\
& \leq C_{3}\left\|x_{k}-x^{*}\right\|^{2}+4 C_{4}\left\|x_{k}-x^{*}\right\|\left\|x_{k-1}-x^{*}\right\|^{2} .
\end{aligned}
$$

It follows from the inequality (15) that the order of convergence (5) is not higher than quadratic. Consequently, there exist a constant $C_{5} \geq 0$ and a positive integer $N$ such that for all $k \geq N$ we have

$$
\left\|x_{k}-x^{*}\right\| \geq C_{5}\left\|x_{k-1}-x^{*}\right\|^{2}
$$

Then from (15) we obtain

$$
\begin{aligned}
\left\|x_{k+1}-x^{*}\right\| & \leq C_{3}\left\|x_{k}-x^{*}\right\|^{2}+4 C_{4}\left\|x_{k-1}-x^{*}\right\|^{2}\left\|x_{k}-x^{*}\right\| \\
& \leq C_{3}\left\|x_{k}-x^{*}\right\|^{2}+4 \frac{C_{4}}{C_{5}}\left\|x_{k}-x^{*}\right\|^{2}=C_{6}\left\|x_{k}-x^{*}\right\|^{2} .
\end{aligned}
$$

From the last estimate it follows the assertion of the corollary.

Assume that $L, M$ and $N$ are constants. Then from Theorem 1 we get results similar to ones, obtained in [13].

Theorem 2. Let $F+G: D \subseteq \mathbb{R}^{n} \rightarrow \mathbb{R}^{m}$ be continuous on an open convex subset $D$, and $F$ is a continuously differentiable function, $\mathrm{G}$ is a continuous function. Suppose that the problem (4) has a solution $x^{*} \in D$, and the inverse operation $\left(A_{*}^{T} A_{*}\right)^{-1}$ exists, such that $\left\|\left(A_{*}^{T} A_{*}\right)^{-1}\right\| \leq B$.

On the subset $D$, the Fréchet derivative $F^{\prime}$ satisfies the radius Lipschitz condition

$$
\left\|F^{\prime}(x)-F^{\prime}\left(x^{\tau}\right)\right\| \leq(1-\tau) L\left\|x-x^{*}\right\|, \quad x^{\tau}=x^{*}+\tau\left(x-x^{*}\right), \quad 0 \leq \tau \leq 1,
$$

the function $G$ has the first- and second-order divided difference, and

$$
\|G(x, y)-G(u, v)\| \leq M(\|x-u\|+\|y-v\|), \quad\|G(u, x, y)-G(v, x, y)\| \leq N\|u-v\|,
$$

for all $x, y, u, v \in D, \rho(x)=\left\|x-x^{*}\right\| ; L, M$ and $N$ are positive constants.

Furthermore,

$$
\left\|F\left(x^{*}\right)+G\left(x^{*}\right)\right\| \leq \eta, \quad\left\|F^{\prime}\left(x^{*}\right)+G\left(x^{*}, x^{*}\right)\right\| \leq \alpha, \quad B[L+2 M] \eta<1
$$

and $\Omega=\Omega\left(x^{*}, r_{*}\right)=\left\{x:\left\|x-x^{*}\right\|<r_{*}\right\} \subseteq D$, where $r_{*}$ is the unique positive zero of the function $q$ given by

$$
\begin{aligned}
q(r) & =B\left(\alpha+(L+2 M) r+4 N r^{2}\right)\left((L / 2+M) r+4 N r^{2}\right) \\
& +B(L+2 M+4 N r) \eta+B\left(2 \alpha+(L+2 M) r+4 N r^{2}\right)\left((L+2 M) r+4 N r^{2}\right)-1 .
\end{aligned}
$$

Then, for $x_{0}, x_{-1} \in \Omega$, the iterative process $\left\{x_{k}\right\}, k=0,1, \ldots$, generated by (5), is well defined, remains in $\Omega$, and converges to $x^{*}$. Moreover, the following error estimates hold for all $k \geq 0$ :

$$
\left\|x_{k+1}-x^{*}\right\| \leq C_{1}\left\|x_{k}-x^{*}\right\|+C_{2}\left\|x_{k}-x_{k-1}\right\|^{2}+C_{3}\left\|x_{k}-x^{*}\right\|^{2}+C_{4}\left\|x_{k}-x^{*}\right\|\left\|x_{k}-x_{k-1}\right\|^{2},
$$

where

$$
\begin{gathered}
C_{1}=g\left(r_{*}\right)(L+2 M) \eta, \quad C_{2}=g\left(r_{*}\right) N \eta, \\
C_{3}=g\left(r_{*}\right)(L / 2+M)\left(\alpha+(L+2 M) r_{*}+4 N r_{*}^{2}\right), \quad C_{4}=g\left(r_{*}\right) N\left(\alpha+(L+2 M) r_{*}+4 N r_{*}^{2}\right), \\
g(r)=B\left[1-B\left(2 \alpha+(L+2 M) r+4 N r^{2}\right)\left((L+2 M) r+4 N r^{2}\right)\right]^{-1} .
\end{gathered}
$$




\section{Numerical results}

In this section, we present the results of the verifying of the theorem's conditions. We use the Euclidean norm $\|x\|=\sqrt{\sum_{i=1}^{n} x_{i}^{2}}$ for $x \in \mathbb{R}^{n}$.

Example 1. Consider the function $F+G: D \subseteq \mathbb{R} \rightarrow \mathbb{R}^{3}$ given by

$$
\begin{gathered}
F(x)+G(x)=\left(\begin{array}{c}
x+\mu \\
\lambda x^{3}+x-\mu \\
\lambda\left|x^{3}-1\right|-\lambda
\end{array}\right) \\
F(x)=\left(\begin{array}{c}
x+\mu \\
\lambda x^{3}+x-\mu \\
0
\end{array}\right), \quad G(x)=\left(\begin{array}{c}
0 \\
0 \\
\lambda\left|x^{3}-1\right|-\lambda
\end{array}\right),
\end{gathered}
$$

where $\lambda, \mu \in \mathbb{R}$ are two parameters.

The unique solution of this problem is $x_{*}=0$. Therefore, we can set constants $\alpha, \eta$ and $B$ as follows: $\eta=\sqrt{2}|\mu|, \alpha=\sqrt{2}, B=1 / 2$.

Let $D=\{x:|x|<1\}$. Then

$$
F^{\prime}(x)=\left(\begin{array}{c}
1 \\
3 \lambda x^{2}+1 \\
0
\end{array}\right), G(x, y)=\left(\begin{array}{c}
0 \\
0 \\
\frac{\lambda\left|x^{3}-1\right|-\lambda\left|y^{3}-1\right|}{x-y}
\end{array}\right)=\left(\begin{array}{c}
0 \\
0 \\
-\lambda\left(x^{2}+x y+y^{2}\right)
\end{array}\right)
$$

and

$$
\begin{aligned}
G(u, x, y) & =\frac{G(u, x)-G(u, y)}{x-y} \\
& =\left(\begin{array}{c}
0 \\
0 \\
\frac{-\lambda\left(u^{2}+u x+x^{2}\right)+\lambda\left(u^{2}+u y+y^{2}\right)}{x-y}
\end{array}\right)=\left(\begin{array}{c}
0 \\
0 \\
-\lambda(u+x+y)
\end{array}\right),
\end{aligned}
$$

and

$$
\begin{gathered}
\left\|F^{\prime}(x)-F^{\prime}\left(x^{\tau}\right)\right\|=3|\lambda|(1-\tau)(1+\tau)|x|\left|x-x^{*}\right| \leq 6|\lambda||x|(1-\tau)\left|x-x^{*}\right| \\
\|G(x, y)-G(u, v)\| \leq|\lambda|(|u+x+y||u-x|+|v+y+u||v-y|) \\
\|G(u, x, y)-G(v, x, y)\| \leq|\lambda||u-v|
\end{gathered}
$$

That is, we can set constants $L=6|\lambda|, M=3|\lambda|, N=|\lambda|$. We consider problems with zero and nonzero residuals. Solving equation (16) for different values of parameters $\lambda, \mu$ we get results, given in Table 1.

\begin{tabular}{|cc|c|c|}
\hline$\lambda$ & $\mu$ & $r_{*}$ & $B[L+2 M] \eta$ \\
\hline 0.35 & 0 & 0.1076417713313384 & 0 \\
0.1 & 0.3 & 0.2676148592421200 & 0.2545584412271572 \\
\hline
\end{tabular}

Table 1. Results for different values of $\lambda$ and $\mu$. 
Let $\lambda=0.35, \mu=0, x_{0}=0.1, y_{0}=0.1001$. In this case we have problem with zero residual. The solution was obtained in 3 iterations.

\begin{tabular}{|c|cc|}
\hline$k$ & $\rho\left(x_{k+1}\right)$ & The right side of $(17)$ \\
\hline 0 & $3.535982373516905 \mathrm{e}-04$ & $8.668044825593081 \mathrm{e}-02$ \\
1 & $2.150913546502143 \mathrm{e}-09$ & $6.156060534217174 \mathrm{e}-06$ \\
2 & $2.067951531382569 \mathrm{e}-24$ & $4.286170529089731 \mathrm{e}-16$ \\
\hline
\end{tabular}

Table 2. The results for $\lambda=0.35, \mu=0$.

Let $\lambda=0.1, \mu=0.3, x_{0}=0.1, y_{0}=0.1001$. In this case we have problem with nonzero residual. The solution was obtained in 3 iterations too.

\begin{tabular}{|c|cc|}
\hline$k$ & $\rho\left(x_{k+1}\right)$ & The right side of $(17)$ \\
\hline 0 & $5.489482150691644 \mathrm{e}-04$ & $6.922777353526340 \mathrm{e}-02$ \\
1 & $1.384555821295347 \mathrm{e}-08$ & $7.884351861069518 \mathrm{e}-04$ \\
2 & $1.301043268727095 \mathrm{e}-17$ & $2.232703312141992 \mathrm{e}-08$ \\
\hline
\end{tabular}

Table 3. The results for $\lambda=0.1, \mu=0.3$.

Therefore, all conditions of Theorem 2 are satisfied. We see that the right sides of estimate (17) for problem with zero residual decreases faster than for problem with nonzero residual (see Tables 2 and 3, respectively). This confirms the convergence orders of the method for different types of problem (4).

\section{Conclusions}

We investigated the local convergence of the Gauss-Newton-Kurchatov method for solving nonlinear least squares problems under generalized Lipshitz conditions. The quadratic convergence order of the method for problems with zero residual is established. Obtained numerical results are consistent with the theoretical ones.

\section{References}

[1] Argyros I.K. Convergence and applications of Newton-type iterations. Springer-Verlag, New York, 2008.

[2] Argyros I.K., Magreñán Á.A. A contemporary study of iterative methods. Convergence, dynamics and applications. Academic Press, London, 2019. doi:10.1016/C2015-0-04301-5

[3] Argyros I.K., Shakhno S. Extended Two-Step-Kurchatov method for solving Banach space valued nondifferentiable equations. Int. J. Appl. Math. Comput. Math. 2020, 6 (2), 32. doi:10.1007/s40819-020-0784-y

[4] Argyros I.K., Shakhno S.M., Yarmola H.P. Extended semilocal convergence for the Newton-Kurchatov method. Mat. Stud. 2020, 53 (1), 85-91. doi:10.30970/ms.53.1.85-91

[5] Argyros I.K., Shakhno S., Yarmola H. Improving convergence analysis of the Newton-Kurchatov method under weak conditions. Comput. 2020, 8 (1), 8. doi:10.3390/computation8010008

[6] Cãtinac E. On some iterative methods for solving nonlinear equations. Rev. Anal. Numér. Théor. Approx. 1994, 23 (1), 47-53.

[7] Dennis J.E.Jr., Schnabel R.B. Numerical methods for unconstrained optimization and nonlinear equations. SIAM, Philadelphia, 1996.

[8] Hernández M.A. Rubio M.J. A uniparametric family of iterative processes for solving nondiffrentiable operators. J. Math. Anal. Appl. 2002, 275 (2), 821-834. doi:10.1016/S0022-247X(02)00432-8 
[9] Hernández-Verón M.A., Rubio M.J. On the local convergence of Newton-Kurchatov-type method for nondifferentiable operators. Appl. Math. Comput. 2017, 304 (1), 1-9. doi:10.1016/j.amc.2017.01.010

[10] Ortega J.M., Rheinboldt W.C. Iterative solution of nonlinear equations in several variables. Acad. Press, San Diego, 1970.

[11] Ren H., Argyros I.K. Local convergence of a secant type method for solving least squares problems. Appl. Math. Comput. 2010, 217 (8), 3816-3824. doi:10.1016/j.amc.2010.09.040

[12] Ren H., Argyros I.K., Hilout S. A derivative free iterative method for solving least squares problems. Numer. Algor. 2011, 58 (4), 555-571. doi:10.1007/s11075-011-9470-9

[13] Shakhno S.M. Gauss-Newton-Kurchatov method for solving nonlinear least squares problems. J. Math. Sci. 2020, 247 (1), 58-72. doi:10.1007/s10958-020-04789-y (translation of Mat. Metody Fiz.-Mekh. Polya 2017,60 (4), 52-62. (in Ukrainian))

[14] Shakhno S.M., Gnatyshyn O.P. Iterative-difference methods for solving nonlinear least-squares problem. In: Arkeryd L., Bergh J., Brenner P., Pettersson R. (Eds.) Progress in Industrial Mathematics at ECMI 98, Teubner, Stuttgart, 1999, 287-294.

[15] Shakhno S.M., Iakymchuk R.P., Yarmola H.P. An iterative method for solving nonlinear least squares problems with nondifferentiable operator. Mat. Stud. 2017, 48 (1), 97-107. doi:10.15330/ms.48.1.97-107

[16] Shakhno S.M., Yarmola H.P., Shunkin Yu.V. Convergence analysis of the Gauss-Newton-Potra method for nonlinear least squares problems. Mat. Stud. 2018, 50 (2), 211-221. doi:10.15330/ms.50.2.211-221

[17] Shakhno S.M., Shunkin Yu.V. One combined method for solving nonlinear least squares problems. Bull. Lviv Univ. Ser. Appl. Math. Inform. 2017, 25, 38-48 (in Ukrainian).

[18] Ulm S. On generalized divided differences, I. Izv. Akad. Nauk ESSR Ser. Fiz. Mat. Tekh. Nauk 1967, 16 (1), 13-26 (in Russian).

[19] Ulm S. On generalized divided differences, II. Izv. Akad. Nauk ESSR Ser. Fiz. Mat. Tekh. Nauk 1967, 16 (2), 146-156 (in Russian).

[20] Wang X. Convergence of Newton's method and uniqueness of the solution of equations in Banach space. IMA J. Numer. Anal. 2000, 20 (1), 123-134. doi:10.1093/imanum/20.1.123

Received 15.04.2020

Revised 26.06.2020

Шахно С.М., Ярмола Г.П. Аокальна збіжність методу Гаусса-Ньютона-Куриатова за узагальнених умов Аіпшиця // Карпатські матем. публ. - 2021. - Т.13, №2. - С. 305-314.

У роботі досліджено локальну збіжність методу Гаусса-Ньютона-Курчатова для розв'язання нелінійних задач про найменші квадрати. Цей метод $є$ комбінацією методів Гаусса-Ньютона та Курчатова і застосовується для задач з декомпозицією оператора. Аналіз збіжності методу проведено за узагальнених умов $\Lambda$ іпшиця. Встановлено умови, радіус та порядок збіжності методу. Наведено чисельні приклади, які підтерджують теоретичні результати.

Ключові слова і фрази: метод Гаусса-Ньютона-Курчатова, локальна збіжність, похідна Фреше, поділена різниця, узагальнена умова $\Lambda$ іпшиця, область збіжності. 